\title{
Müllerianosis with Intestinal Metaplasia: A Case Report
}

\author{
Sofía DEL CARMEN, Marta RODRíGUEZ, M. Asunción GÓMEZ, Miguel Ángel CRUZ, M. Antonia NÚÑEZ, \\ Magdalena SANCHO
}

Complejo Asistencial de Salamanca, Anatomía Patológica, SALAMANCA, SPAIN

\begin{abstract}
Müllerianosis or Müllerian choristomas are developmental alterations that consist of an organoid structure with normal Müllerian tissue. We present a 62-year-old patient diagnosed on ultrasound scanning and on CT scan of bilateral ovarian cysts. During surgery, a left ovarian cyst and retroperitoneal tumor (adhered to sigmoid serous surface) were found. On histological examination, the tumor corresponded with a Müllerian choristoma showing endometrial, endosalpingeal and endocervical epithelium, with foci of intestinal metaplasia, a phenomenon not described in the literature.
\end{abstract}

Key Words: Choristoma, Metaplasia, Gynecologic disease

\section{INTRODUCTION}

Müllerianosis was first defined by Batt as an organoid structure with the presence of rests of Müllerian tissue (endometrial, endosalpingeal or endocervical tissue) located in the pelvic peritoneal cavities. As Müllerian tissue had been described in different locations, the term Müllerianosis was redefined (by Batt) as a choristoma with the presence of Müllerian tissue including one, two or three normal components, in any location. This ectopic tissue is thought to be incorporated within normal organs during embryologic development (1).

We present a case of pelvic Müllerianosis with endometriosis, endosalpingiosis and endocervicosis with foci of intestinal metaplasia within the endocervical epithelium, a phenomenon not described in the literature.

\section{CASE REPORT}

We present a 62-year-old woman, with no relevant clinical or surgical history, who consulted her family doctor and was referred for a painful tumor in the left inguinal region for two months. Abdominal ultrasound revealed a bilateral cystic tumor of adnexal origin. MR scan described two big pelvic adnexal masses that were cystic, separated, well delimited and with no evidence of solid components inside. Those lesions showed enhancement of the walls after contrast administration (Figure 1). Tumor markers levels in serum were normal for CA125, CA15.3 and alpha fetoprotein, showing a slight elevation of CA19.9 (35.4 units/ml).

Received : 31.03.2015 Accepted : 10.06.2015
The patient underwent total abdominal hysterectomy with clinical and radiological suspicion of uterine leiomyomas and bilateral ovarian tumors. During the operation, a cystic tumor on the left ovary was excised, and a retroperitoneal cystic tumor adherent to the sigmoid wall, with no relation with the uterus or the right ovary was found. Surgeons performed a hysterectomy with bilateral oophorectomy and resection of the pelvic mass.

Macroscopically, there was a cystic tumor of $10 \times 7 \times 5 \mathrm{~cm}$ on the left ovary. The external surface was smooth and whitish, with marked vascularity. The internal surface was granular but with no nodular nor papillary formations. The fallopian tube did not show any macroscopic alteration. On histological examination, the cystic lesion was a mucinous cystadenoma and on the fallopian tube there were foci of mucosal tubal endometriosis.

The right ovary was macroscopically normal and sized $2 \mathrm{x} 1$ $\mathrm{cm}$, and in the image it was received attached to the uterus. The uterine wall showed several subserosal and intramural formations. The biggest one was $3 \mathrm{~cm}$ in diameter. These tumors were well delineated, round, and on cut surface they were firm, gray-white and whorled. On histological sections these lesions were confirmed as leiomyomas. Foci of adenomyosis were also found on the uterus.

The pelvic tumor was $13 \times 8 \times 3 \mathrm{~cm}$ in size. It had elastic consistency and the surface was white and smooth. On cut surface its appearance was heterogeneous, alternating cystic cavities of different diameters with aqueous content

Correspondence: Sofía DEL CARMEN

Complejo Asistencial de Salamanca, Anatomía Patológica,

SALAMANCA, SPAIN

E-mail: sofia_delcarmen@hotmail.com Phone: + 34923260102 


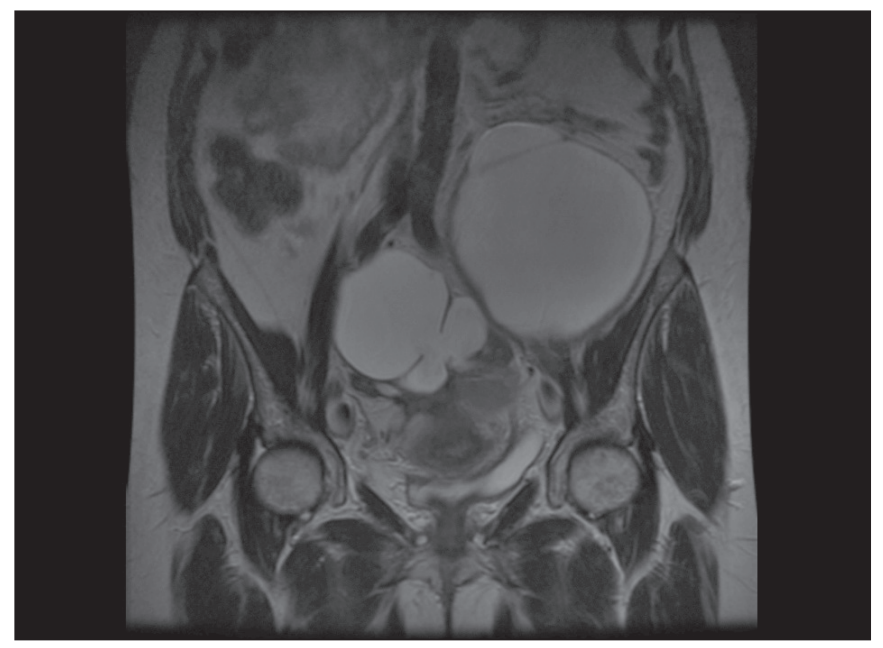

Figure 1: MR scan image showing two pelvic adnexal masses cystic, septated, well delimited and with no evidence of solid components inside.

and solid fibrous areas. The internal surface of the cysts was rough showing zones with papillary formations (Figure $2 \mathrm{~A}$ ). The microscopic examination (Figure 2B) revealed endosalpingeal (Figure 2C), endometrial and endocervical epithelium covering the walls of the cystic cavities. The epithelial cells lining the cysts showed no atypia and no signs of infiltration was observed. The walls of cavities showed muscular hyperplasia. Foci of goblet cells with no cytological atypia were found within the areas of endocervical mucosa (Figure 2D). On immunohistochemical study the distinct müllerian epithelium were positive for CK7 (Figure 2E), estrogen receptors and progesterone receptors, and they were negative for CK20 and CEA. Goblet cells were positive for CK20 (Figure 2F) and CDX2 (Figure 2G), which confirmed these cells as intestinal metaplastic epithelium.

With all findings described, our final diagnosis was Müllerian choristoma (or pelvic Müllerianosis) with endosalpingiosis, endometriosis and endocervicosis with foci of intestinal metaplasia. The patient did not show any important complication after surgery, and at the present time, after 4 months of follow-up she is free of disease.

\section{DISCUSSION}

Choristomas were defined in 1904 by Eugen Albrecht as tumors composed of histologically normal tissues misplaced within organs during organogenesis (2). Those composed of endometrial, endosalpingeal and endocervical tissues are Müllerian choristomas.

Over the years, the term «Müllerianosis» has been reviewed several times. First, in 1989, Batt defined Müllerianosis as an organoid structure with the presence of remnants of Müllerian tissue located on pelvic peritoneal cavities (rectovaginal space, posterior broad ligament and pararectal space), accepting for its diagnosis the presence of, at least, one segment of Müllerian-derived epithelium (endometrial, endosalpingeal and/or endocervical tissue) (3).

As Müllerian tissue had been described in different locations such as the urinary bladder (4), the liver, appendix (5), skin (6), paravertebral (7), etc., in 1996, Young and Clement proposed stricter histological criteria, and postulated that Müllerianosis were lesions in any site containing endometriosis, endosalpingiosis and endocervicosis. They proposed that for an accurate diagnosis of Müllerianosis, two or three tissue types should be found (8). Later in 2007, Batt redefined the term "Müllerianosis" as a choristoma with the presence of Müllerian tissue including one, two or three normal components, in any location (1).

There are 4 Müllerian disorders named as endometriosis, endosalpingiosis, adenomyosis and endocervicosis, and they can be acquired or developmental (9). It is important to distinguish between developmental and acquired Müllerianosis, due to the fact that both entities are characterized on histological examination by the presence of misplaced normal Müllerian tissue, but their pathogenesis and location in organs is different.

Acquired endometriosis is viewed as endometrial tissue that invades the outer surface of organs. Several theories try to explain the mechanism of endometriosis such as surgery, retrograde menstruations, hematogenous metastasis or coelomic metaplasia, which are some examples (10). In acquired endosalpingiosis there is endosalpingeal tissue invading the surface of organs. Endometrial glands and stroma invade the myometrium in acquired adenomyosis. Acquired endocervicosis is a rare lesion described after hysterectomy, due to transplantation of endocervical tissue (9).

Developmental Müllerianosis consists of Müllerian tissue misplaced within organs, in any location. It is a structure of embryonic origin, and it is sometimes associated with other malformations or congenital abnormalities. The fact that it has been found in female fetuses, infants, children, adolescents and adults supports the theory that it is a congenital developmental disease (11). The etiology of this lesion still remains unknown.

According to Batt's criteria (1), developmental Müllerianosis can be diagnosed with certainty when there is no evidence of pelvic endometriosis, there are no direct communications with the endocervix, endometrium or endosalpinx, and there is no history of surgery on the reproductive organs. 


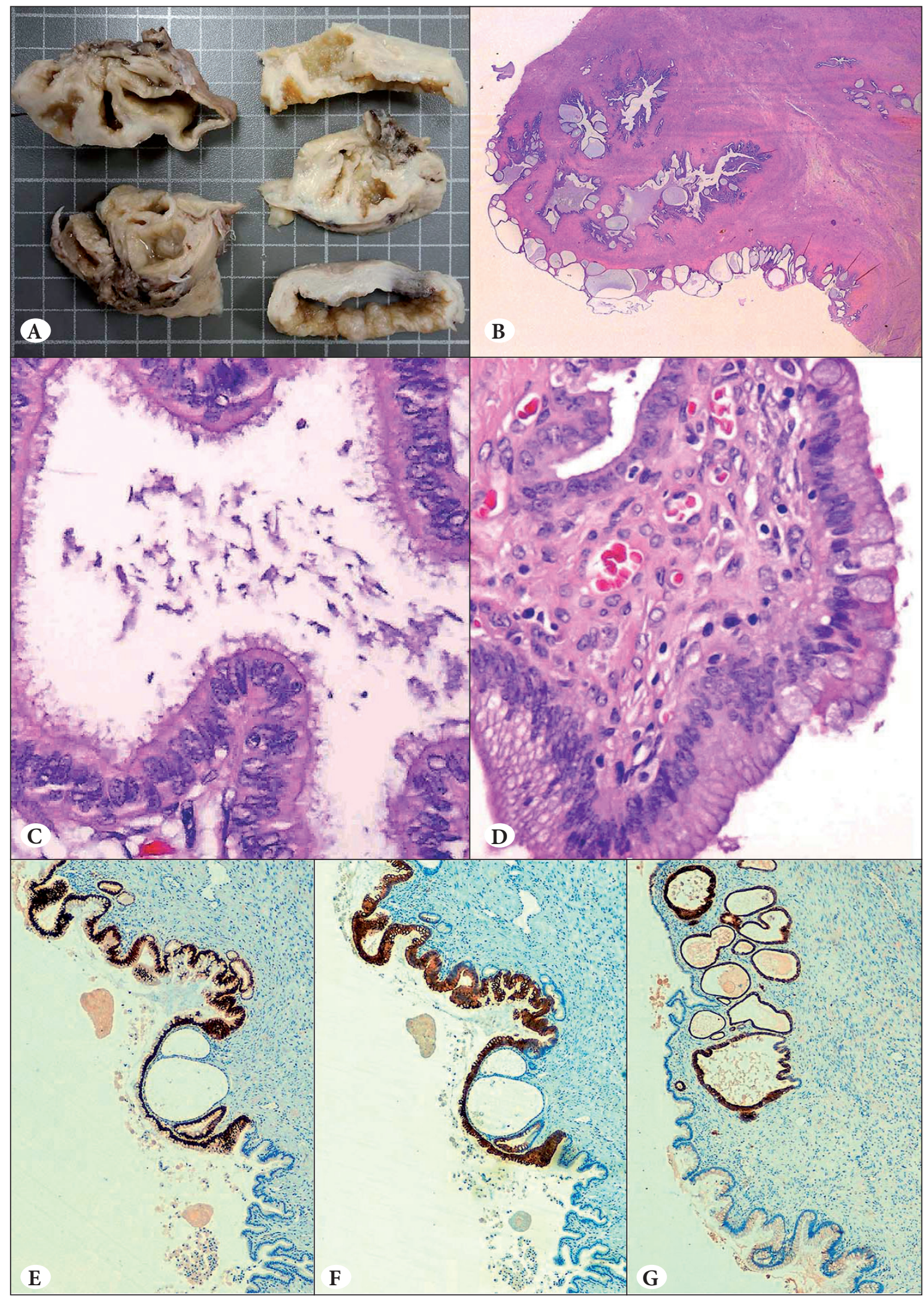

Figure 2: Pelvic tumor: A) Macro image showing a heterogeneous cut surface, alternating cystic cavities with papillary and rough walls and solid fibrous areas. B) Micro image (panoramic). C) Endosalpingeal epithelium (HxE; x400). D) Goblet cells, with no cytological atypia, within the areas of endocervical mucosa (HxE; x400). E) Goblet cells positive for CDX2 (CDX2; x40). F) Goblet cells positive for CK20 (CK20; x40). G) Müllerian epithelium positive for CK7 (CK7; x40). 
The diagnosis of a Müllerian choristoma requires both clinical and histological criteria.

In our case, the patient had no history of abdominal surgery, no evidence of pelvic endometriosis and the lesion attached to sigmoid wall (on its serosal surface), without relation to the uterus or ovary. Taking this into account, the pelvic lesion we have described meets Batt's criteria for considering it a developmental Müllerianosis. In our review of the literature we have not found any similar finding. In our case we found foci of intestinal metaplasia within areas of endocervical epithelium that expressed CK20 and CDX2. We do not consider this intestinal epithelium as intestinal contamination since the lesion was related to the serosal surface, with no connection with the intestinal mucosa.

In our case there were no signs of malignancy in the foci of intestinal metaplasia. We consider this finding a consequence of mechanical traction suffered by the tumor, since we could consider it a congenital lesion as the patient did not have previous surgery, and for its location in the pelvic cavity.

Clinically, Müllerian choristomas may be asymptomatic or they can be associated with pelvic pain, infertility, or weakness due to bleeding if they contain endometrium (1). There are some described cases of Müllerianosis that became malignant, but this is not the rule (12). Surgical excision is considered curative, with no need of chemotherapy or radiotherapy, and no recurrence is expected. Our case is free of disease after 4 months of follow-up.

\section{REFERENCES}

1. Batt RE, Smith RA, Buck Louis GM, Martin DC, Chapron C, Koninckx PR, Yeh J. Müllerianosis. Histol Histopathol. 2007;22:1161-6.

2. Ober WB. Selected items from the history of pathology: Eugen Albrecht, MD (1872-1908): Hamartoma and choristoma. Am J Pathol. 1978;91:606.

3. Batt RE, Smith RA. Embryologic theory of histogenesis of endometriosis in peritoneal pockets. Obstet Gynecol Clin North Am. 1989;16:15-28.

4. Guan H, Rosenthal DL, Erozan YS. Mullerianosis of the urinary bladder: Report of a case with diagnosis suggested in urine cytology and review of literature. Diagn Cytopathol. 2012;40:9971001.

5. Cajigas A, Axiotis CA. Endosalpingiosis of the vermiform appendix. Int J Gynecol Pathol. 1990;9:291-5.

6. Hung T, Yang A, Binder SW, Barnhill RL. Cutaneous ciliated cyst on the finger: A cutaneous mullerian cyst. Am J Dermatopathol. 2012;34:335-8.

7. Batt RE, Mhawech-Fauceglia P, Odunsi K, Yeh J. Pathogenesis of mediastinal paravertebral müllerian cysts of Hattori: Developmental endosalpingiosis-müllerianosis. Int J Gynecol Pathol. 2010;29:546-51.

8. Young RH, Clement PB. Müllerianosis of the urinary bladder. Mod Pathol. 1996;9:731-7.

9. Batt RE, Yeh J. Müllerianosis: Four developmental (embryonic) mullerian diseases. Reprod Sci. 2013;20:1030-7.

10. Sampson JA. Heterotopic or misplaced endometrial tissue. Am J Obs Gynecol. 1925;10:649-64.

11. Signorile PG, Baldi F, Bussani R, D’Armiento M, De Falco M, Baldi A. Ectopic endometrium in human foetuses is a common event and sustains the theory of müllerianosis in the pathogenesis of endometriosis, a disease that predisposes to cancer. J Exp Clin Cancer Res. 2009;28:49.

12. Kruse A-J, Slangen B, Dunselman GA, Pirens T, Bakers FC, Baak JP, de Vijver KV. Neoplastic transformation of endocervicosis into an extraovarian mucinous cystadenocarcinoma. Hum Pathol. 2011;42:743-8. 